Overcoming the diffraction limit induced by microsphere optical nanoscopy

This content has been downloaded from IOPscience. Please scroll down to see the full text. 2013 J. Opt. 15125710

(http://iopscience.iop.org/2040-8986/15/12/125710)

View the table of contents for this issue, or go to the journal homepage for more

Download details:

IP Address: 130.88.106.243

This content was downloaded on 11/11/2013 at 13:57

Please note that terms and conditions apply. 


\title{
Overcoming the diffraction limit induced by microsphere optical nanoscopy
}

\author{
Seoungjun Lee ${ }^{1}$, Lin $\mathrm{Li}^{1}$, Yacob Ben-Aryeh ${ }^{2}$, Zengbo Wang ${ }^{1,3}$ and \\ Wei Guo ${ }^{1}$ \\ ${ }^{1}$ School of Mechanical, Aerospace and Civil Engineering, University of Manchester, Manchester \\ M13 9PL, UK \\ 2 Physics Department, Technion-Israel Institute of Technology, Haifa 32000, Israel \\ ${ }^{3}$ Schools of Electronics, Bangor University, Dean Street, Bangor, Gwynedd LL57 1UT, UK \\ E-mail: seoungjun.lee@postgrad.manchester.ac.uk
}

Received 29 May 2013, accepted for publication 1 October 2013

Published 24 October 2013

Online at stacks.iop.org/JOpt/15/125710

\begin{abstract}
The microsphere optical nanoscopy (MONS) technique recently demonstrated the capability to break the optical diffraction limit with a microsphere size of 2-9 $\mu \mathrm{m}$ fused silica. We report that larger polystyrene microspheres of 30,50 and $100 \mu \mathrm{m}$ diameters can overcome the diffraction limit in optical imaging. The sub-diffraction features of a Blu-ray Disc and gold nano-patterned quartz were experimentally observed in air by coupling the microspheres with a standard optical microscope in the reflected light illumination mode. About six to eight times magnification was achieved using the MONS. The mechanism of the MONS was theoretically explained by considering the transformation of near-field evanescent waves into far-field propagating waves. The super-resolution imaging was demonstrated by experiments and theoretical simulations.
\end{abstract}

Keywords: super resolution, diffraction limit, nanoscopy

(Some figures may appear in colour only in the online journal)

\section{Introduction}

The spatial resolution of conventional optical microscopy is restricted by the diffraction limit, which prevents the imaging of sub-wavelength objects with dimensions less than $200 \mathrm{~nm}$. Super-resolution imaging can be achieved by the capture of near-field evanescent waves which are diffraction unlimited [1]. The perfect superlens is well known to overcome the Abbe diffraction limit to use a negative index medium which can restore an evanescent wave [2]. The near-field superlens resolves sub-diffraction-limited imaging using a negative index metamaterial [3, 4]. With the plane metamaterial superlenses, the evanescent wave is coupled with the propagating wave in the far-field without magnification [5, 6]. From the introduction of hyperlenses using metamaterials and curved lens geometry, magnified sub-diffraction-limited objects were observed by converting the evanescent wave into a propagating wave [7]. The hyperlens was experimentally demonstrated at visible wavelengths using nine pairs of silver and titanium oxide metamaterial layers and gold multilayer photonic crystals $[8,9], 36$ brass fins for the acoustic wave [10], and 16 half-cylindrical layers of gold and aluminum oxide on quartz at $365 \mathrm{~nm}$ wavelength [11]. Super-resolution imaging by the use of the microsphere optical nanoscopy (MONS) technique was demonstrated using a fused silica microsphere of $4.74 \mu \mathrm{m}$ diameter combined with a conventional optical microscope to resolve $50 \mathrm{~nm}$ objects with a white light source [12].

\section{Theory and experimental procedure}

We show that the MONS technique can increase the resolution beyond the Abbe limit by transforming evanescent waves to propagating waves. The mechanism can be related to the Helmholtz equation [13] in a homogeneous medium:

$$
k_{0}^{2}=k_{x}^{2}+k_{y}^{2}+k_{z}^{2}
$$


where $k_{0}=(2 \pi n) / \lambda, n$ is the refractive index, and $k_{x}, k_{y}, k_{z}$ are the wavevector components. The evanescent wave can be obtained when

$$
\left(k_{x}^{2}+k_{y}^{2}\right)>k_{0}^{2}
$$

$k_{z}$ becomes imaginary as a decay of the wave in the $z$ direction. The resolution is improved when the components of the wavevector $\vec{k}$ in the $x$ and $y$ planes are increased, and the value of $\lambda$ is decreased. As a result, the evanescent wave is decayed simultaneously. However, the MONS can solve such the limitation of evanescent waves by transforming evanescent waves into propagating waves. In the MONS, the electromagnetic (EM) field $(E(x, y))$ is adjusted by the imaging plane beyond a microsphere. It is transformed by

$$
E^{\prime}(x, y)=E(x, y) G(x, y)
$$

where the transformation functions $G(x, y)$ and $E^{\prime}(x, y)$ can be calculated by geometric optics and the imaging plane of a microsphere. $G(x, y)$ represents the conversion of the imaging plane from the object's plane to a special plane beyond a microsphere. The super-resolution effect can be explained by the analysis of the spatial wavevector coordinates. $G(x, y)$ can be expressed by Fourier transformation into the wavevector spatial coordinates as [14]

$$
G\left(k_{x}, k_{y}\right)=\int_{-\infty}^{\infty} \int_{-\infty}^{\infty} G(x, y) \exp \left[-\mathrm{i}\left(k_{x} x+k_{y} y\right)\right] \mathrm{d} x \mathrm{~d} y .
$$

The Fourier amplitude $A\left(k_{x}, k_{y}\right)$ is determined by the Fourier inverse of $E(x, y)$, which is given by

$$
A\left(k_{x}, k_{y}\right)=\int_{-\infty}^{\infty} \int_{-\infty}^{\infty} E(x, y) \exp \left[-\mathrm{i}\left(k_{x} x+k_{y} y\right)\right] \mathrm{d} x \mathrm{~d} y
$$

where $k_{x}, k_{y}$ are the wavevectors in the $x$ and $y$ directions. $E^{\prime}(x, y), G(x, y)$ and $E(x, y)$ are given by equations (3), (4) and (5) respectively. $E^{\prime}\left(k_{x}, k_{y}\right)$ can be obtained by

$$
\begin{aligned}
E^{\prime}\left(k_{x}, k_{y}\right)= & \int_{-\infty}^{\infty} \int_{-\infty}^{\infty} A\left(k_{x}^{\prime}, k_{y}^{\prime}\right) \\
& \times G\left(k_{x}-k_{x}^{\prime}, k_{y}-k_{y}^{\prime}\right) \mathrm{d} k_{x}^{\prime} \mathrm{d} k_{y}^{\prime} .
\end{aligned}
$$

The convolution of the spatial wavevector transfer functions $G\left(k_{x}, k_{y}\right)$ and $A\left(k_{x}, k_{y}\right)$ can cause the spreading of the spatial wavevector. Thus, the evanescent wave in the object plane can be transformed by convolution to the propagating wave. The spreading of the wavevector could be significantly affected by $G\left(k_{x}, k_{y}\right)$ with very wide $\left(k_{x}, k_{y}\right)$ coordinations.

A plane electromagnetic field incident perpendicular to a flat and smooth metallic boundary plane is refractive as a homogeneous reflective plane wave. In the MONS, the object plane is curved at certain points where the object has a concave and convex surface. From the convex surface the reflective EM beam is diverging and thus decreasing the EM field near such points, while from the concave surface the reflective EM is converging and thus increasing the EM field intensity near these points. The reflective EM field near the object plane has variable EM field intensity from one to one correspondence of the object curvature properties. A similar effect occurs for the transmitted EM field through the object

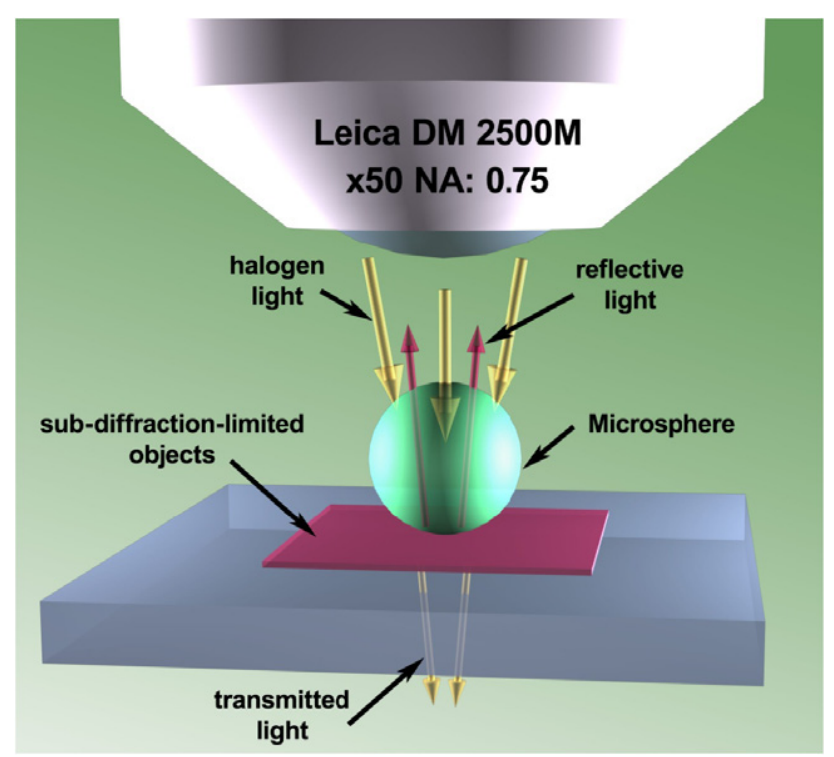

Figure 1. Schematic diagram of the MONS technique for super-resolution imaging. The MONS consists of a microsphere, and a standard optical microscope at the reflective mode illuminated with a halogen light source.

plane where the convex and concave surface affects the EM field distribution near the object plane. The concave surface leads to convergence of the transmitted EM beam while the convex surface leads to beam divergence. Such effects lead to EM imaging of the object including their fine structure, which can be much smaller than a wavelength. Thus, the super-resolution imaging is achieved by such EM waves collected by the microsphere which absorb evanescent waves and transform them into propagating waves.

A small microsphere can limit the view of imaging in a standard optical microscope so the whole view of an object can be sometimes not obtainable or the entire view of the image can be disturbed by the edge of a microsphere. To improve this, larger polystyrene microspheres (up to $100 \mu \mathrm{m}$ diameter) were studied in the present investigation. The effect of sphere size was examined by both experiments and numerical simulations using Mie theory calculation.

In this experiment, 30, 50 and $100 \mu \mathrm{m}$ diameter polystyrene (PS) microspheres were combined with a standard optical microscope (Leica DM $2500 \mathrm{M}$ ) with a $50 \times$ NA 0.75 objective lens in air, and reflective illumination with a halogen light source as shown in figure 1 . The refractive index of the PS microsphere is approximately 1.59 in the visible spectrum. The sub-diffraction-limited objects used in the experiment were a Blu-ray Disc and gold nano-patterned quartz. The Blu-ray Disc has periodic lines of $120 \mathrm{~nm}$ spacing (dark colour) and $180 \mathrm{~nm}$ convex objects (bright colour) in figure 2(a). The gold nano-patterned quartz includes $150 \mathrm{~nm}$ diameter spots (bright colour) and $600 \mathrm{~nm}$ spacing (dark colour) in figure 2(b). The microspheres were spread on the target surface through water drops, and were left to dry. Then the optical microscope was used for observation of targets through the microspheres. The optical imaging of the three different microsphere sizes was recorded and compared with the SEM 


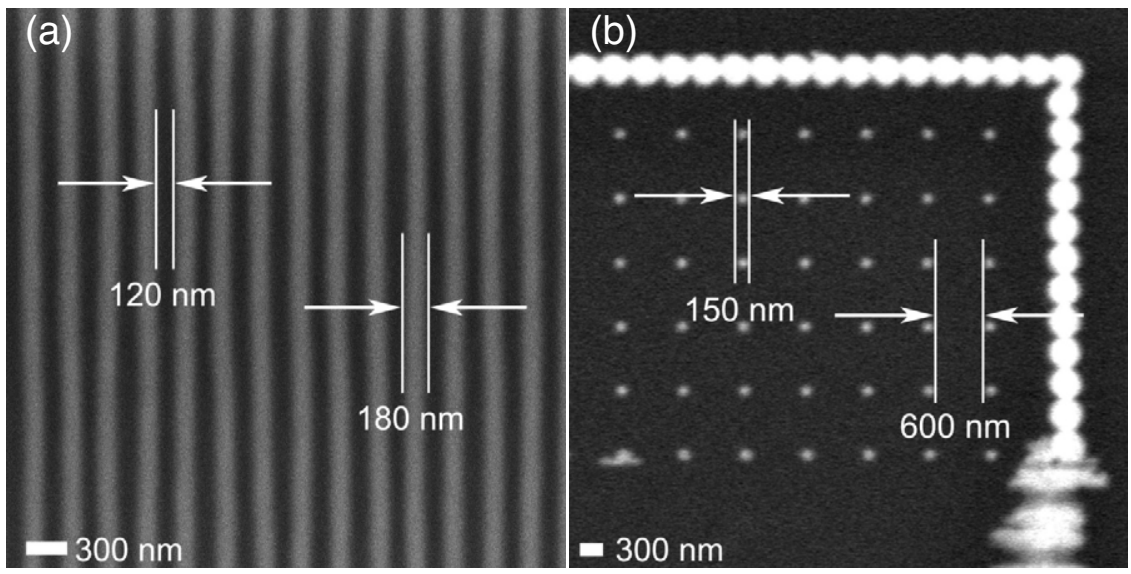

Figure 2. Scanning electron microscope (SEM) images of (a) Blu-ray Disc and (b) gold nano-patterns on quartz. The Blu-ray Disc consists of $180 \mathrm{~nm}$ lines and $120 \mathrm{~nm}$ spacing. The gold nano-patterns on the quartz have $150 \mathrm{~nm}$ spots with $600 \mathrm{~nm}$ spacing.
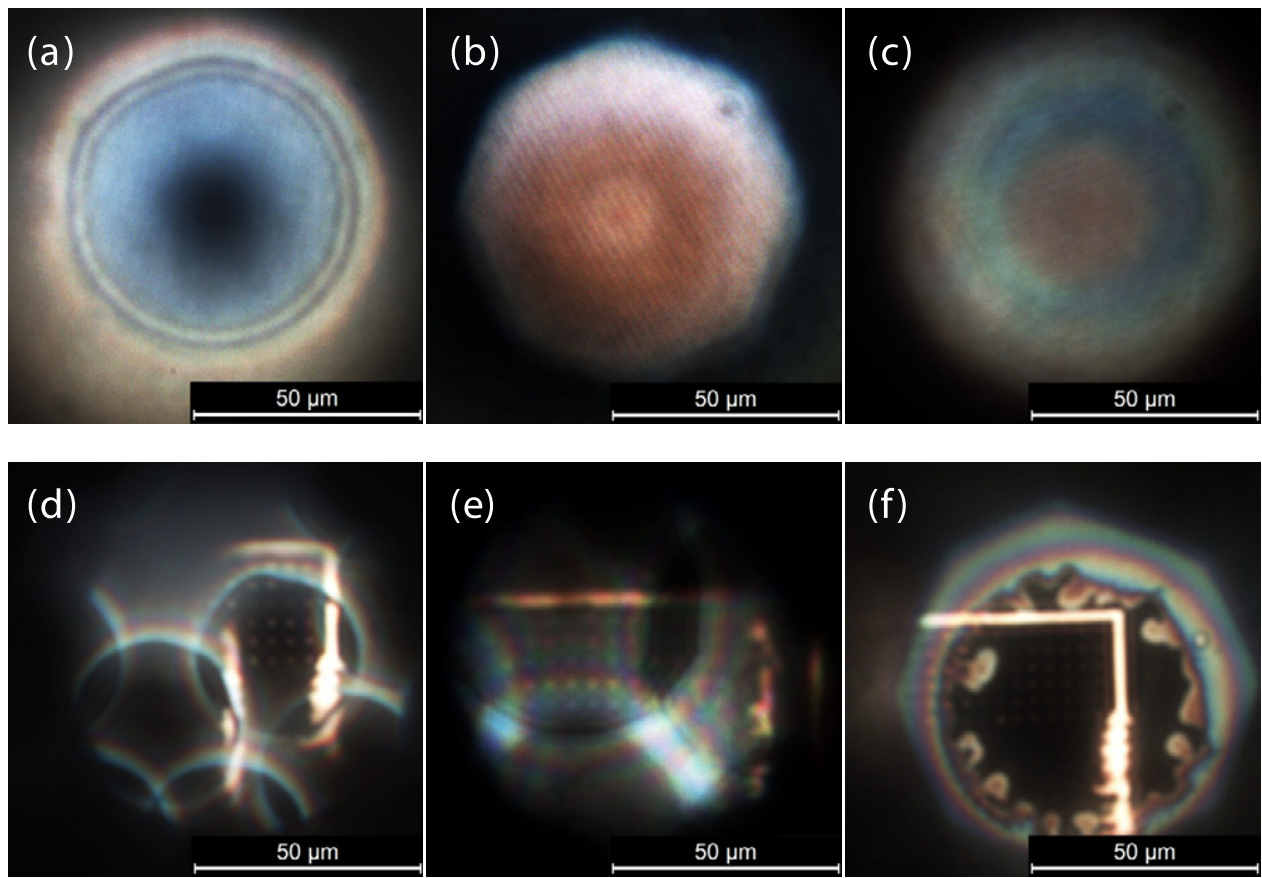

Figure 3. Magnified optical images with 30, 50, and $100 \mu \mathrm{m}$ diameter PS microspheres in air. The Blu-ray Disc was resolved with (a) $30 \mu \mathrm{m}$, (b) $50 \mu \mathrm{m}$ and (c) $100 \mu \mathrm{m}$ PS microspheres when the optical microscope was focused $99 \mu \mathrm{m}, 120 \mu \mathrm{m}$ and $300 \mu \mathrm{m}$ below the target surface, respectively. The gold nano-patterned quartz was observed with the (d) $30 \mu \mathrm{m}$, (e) $50 \mu \mathrm{m}$ and (f) $100 \mu \mathrm{m}$ PS microspheres $60 \mu \mathrm{m}, 140 \mu \mathrm{m}$ and $200 \mu \mathrm{m}$ below the target surface, respectively.

images. The focal image position of the optical microscope was varied to obtain the sharpest images in each case.

\section{Results and discussion}

The minimum resolution $(d)$ of the optical microscope with the 50× NA 0.75 objective lens can be calculated with the known NA and wavelength $\left(\lambda_{0}\right)$, as $d=0.61 \times$ $\left(\lambda_{0} / \mathrm{NA}\right)$. The minimum resolution of the optical microscope is around $300 \mathrm{~nm}$ in the visible spectrum. The sub-wavelength objects of the Blu-ray Disc and the gold nano-patterned quartz were experimentally observed with the 30,50 and $100 \mu \mathrm{m}$ PS microspheres in air as shown in figure 3. These diffraction-limited objects could be not observed without the microspheres in the optical microscope.

The focal image positions and magnifications for the three different sizes of the PS microspheres are shown in table 1. Five pitches were examined to generate the average value of one pitch. One pitch includes the width of one convex line and space for the Blu-ray Disc, and the width of one spot and space for the gold nano-patterned quartz. In SEM calibration, the width of one pitch was $300 \mathrm{~nm}$ for the Blu-ray Disc and $750 \mathrm{~nm}$ for the gold nano-patterned quartz. For the Blu-ray Disc imaging, the sharpest and the best contrast images were observed when the optical microscope was focused $99 \mu \mathrm{m}, 120 \mu \mathrm{m}$ and $300 \mu \mathrm{m}$ 
Table 1. Experimental magnifications and focal image positions of the Blu-ray Disc and the gold nano-patterns on the quartz.

\begin{tabular}{llllllll}
\hline & \multicolumn{3}{c}{ Blu-ray Disc } & & \multicolumn{3}{c}{ Gold nano-patterns on quartz } \\
\cline { 2 - 4 } \cline { 6 - 7 } & $30 \mu \mathrm{m}$ & $50 \mu \mathrm{m}$ & $100 \mu \mathrm{m}$ & $30 \mu \mathrm{m}$ & $50 \mu \mathrm{m}$ & $100 \mu \mathrm{m}$ \\
\hline Magnification & About & About & About & About & About & About \\
& 6.5 & 6.5 & 8.0 & & 5.0 & 7.5 & 5.0 \\
Focal image position & 99 & 120 & 300 & 60 & 140 & 200 \\
$(\mu \mathrm{m})$ & & & & & & \\
\hline
\end{tabular}

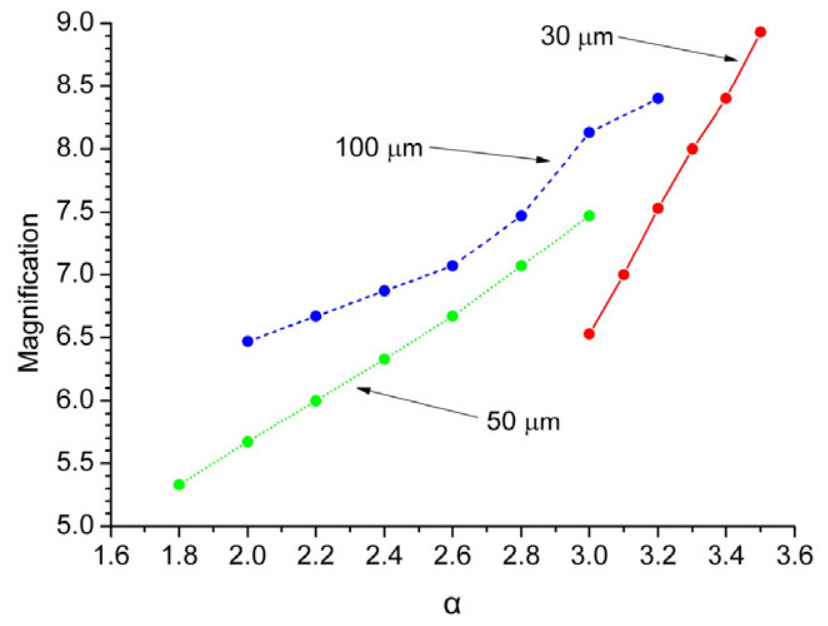

Figure 4. Magnification factor analysis of PS microspheres in the Blu-ray Disc. $\alpha$ is defined as the focal image position below the target substrate divided by the diameter of the PS microsphere. The circle spots are sampling positions for super-resolution imaging. The red solid line, green dotted line and blue dashed line are for $30 \mu \mathrm{m}, 50 \mu \mathrm{m}$ and $100 \mu \mathrm{m}$ PS microspheres, respectively. below the target surface for the $30 \mu \mathrm{m}, 50 \mu \mathrm{m}$ and $100 \mu \mathrm{m}$ PS microspheres, respectively. The ratios of convex (light colour) and spacing (dark colour) surface were kept in the PS microspheres. The black spot was observed in the centre of the $30 \mu \mathrm{m}$ microsphere imaging. The $30 \mu \mathrm{m}$ microsphere might not support sufficient energy to cover the magnified area in the Blu-ray Disc so the diffraction-limited feature cannot easily reach the optical microscope with the $30 \mu \mathrm{m}$ microsphere. For the 30 and $50 \mu \mathrm{m}$ microspheres, overlapped magnified images were observed by the interference between neighbouring microspheres. It can disturb the super-resolution imaging. However, the $100 \mu \mathrm{m}$ microsphere can avoid this interference effect because the view window of the microsphere is larger than the view window of the optical microscope, so the interference cannot reach neighbouring microspheres. In the gold nano-patterned quartz, the sharpest images were observed at $60 \mu \mathrm{m}, 140 \mu \mathrm{m}$ and $200 \mu \mathrm{m}$ below the target surface for the $30 \mu \mathrm{m}, 50 \mu \mathrm{m}$ and $100 \mu \mathrm{m}$ PS microspheres, respectively. The magnified images of the gold-coated spots were unusually expanded, hence the ratio of the spot and space did not hold compared with the SEM image. It may cause a strong plasmon effect of the gold-coated
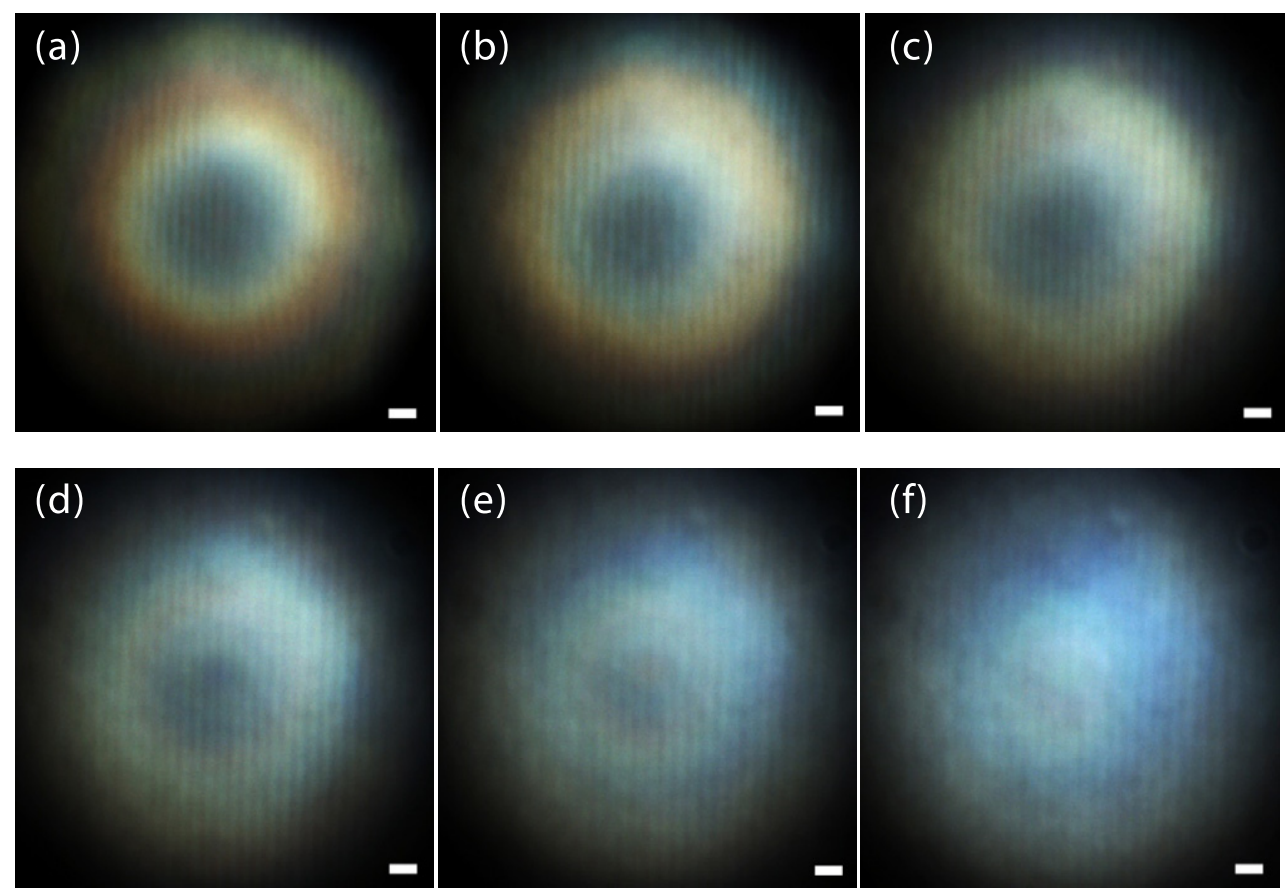

Figure 5. Focal image positions in magnified optical images of the Blu-ray Disc. The images are obtained with $100 \mu \mathrm{m}$ PS microspheres below (a) $220 \mu \mathrm{m}$, (b) $240 \mu \mathrm{m}$, (c) $260 \mu \mathrm{m}$, (d) $280 \mu \mathrm{m}$, (e) $300 \mu \mathrm{m}$ and (f) $320 \mu \mathrm{m}$ positions, respectively. The scale bar is $5 \mu \mathrm{m}$. 

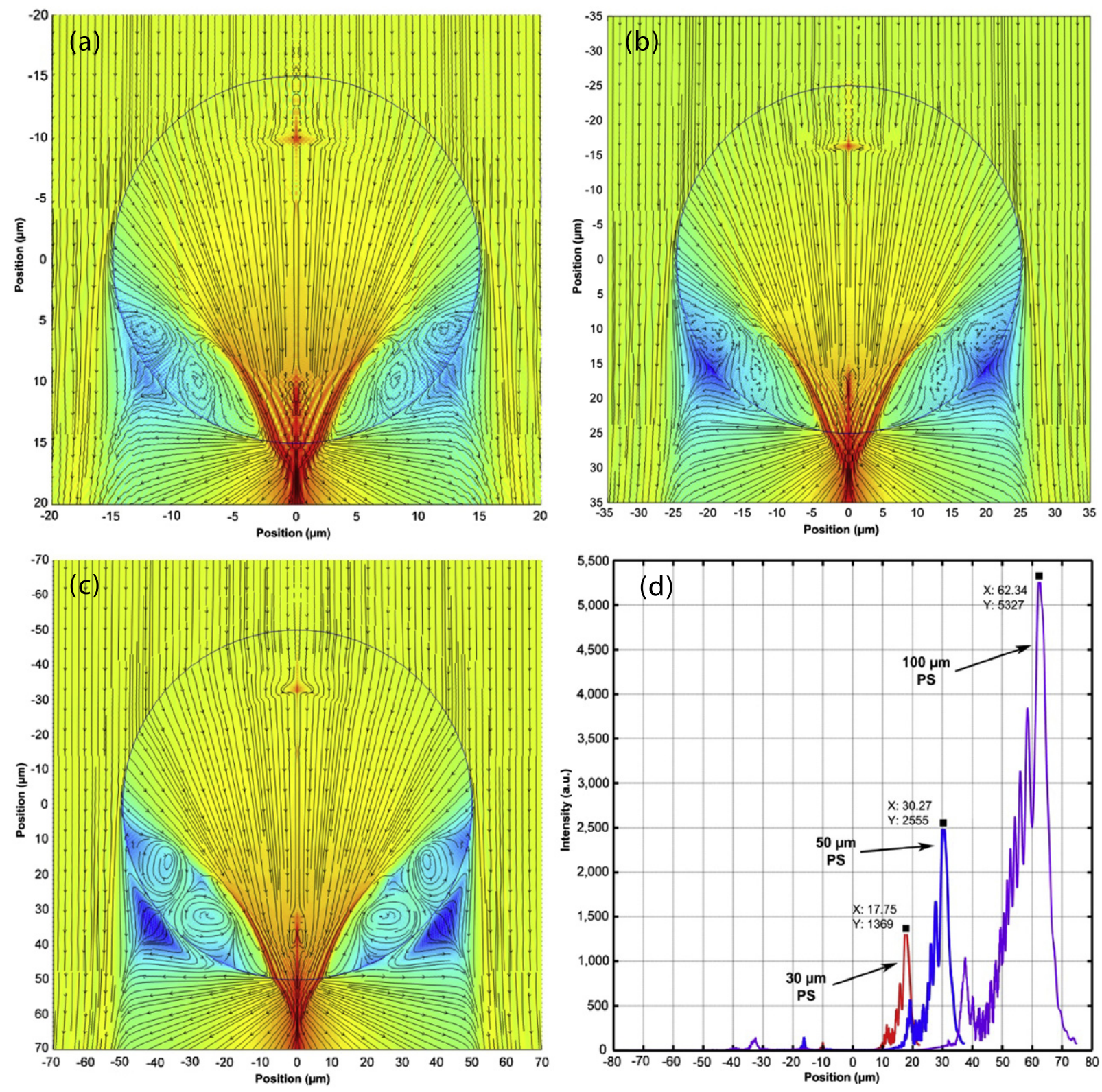

Figure 6. Simulation of the Poynting vector streamlines and the intensity positions at $600 \mathrm{~nm}$ wavelength. The Poynting vector streamlines of (a) $30 \mu \mathrm{m}$, (b) $50 \mu \mathrm{m}$ and (c) $100 \mu \mathrm{m}$ PS microspheres are simulated, and the colour difference indicates the intensity change. The red and blue colours have high and low intensity, respectively. (d) Focal positions of $30 \mu \mathrm{m}$ (red), $50 \mu \mathrm{m}$ (blue) and $100 \mu \mathrm{m}$ (purple) PS microspheres are calculated in a two-dimensional field in the $x z$ plane. The highest intensity positions are $17.75 \mu \mathrm{m}, 30.27 \mu \mathrm{m}$ and $62.34 \mu \mathrm{m}$, respectively. Intensity energy was recorded in the $z$ plane at $x=0$. The incident light source transmits from top to bottom.

surface, which can contribute this distortion. As a result, the surface of the gold-coated spots can be covered by the plasmon so the expanded spots are observed in the optical microscope. Moreover, the black spot was not observed in the gold nano-patterned quartz because the only region of the gold nano-patterns is delivered to the microsphere and the transparent quartz substrate does not disturb the imaging at the same time. Hence, the essential energy of imaging can be obtained in the surface of the gold nano-patterned quartz. Otherwise, the near-field information of the Blu-ray substrate is delivered to the microsphere so the sub-diffraction-limited feature of the Blu-ray can be disturbed by the imaging of the substrate. Therefore, the focal image position and this range can be affected by the substrate conditions. It was also experimentally confirmed that the field of view $(\mathrm{FoV})$ and aperture in the optical microscope can significantly affect image resolution but the magnification was not affected. The value 1 of FoV and the smallest aperture was optimum to give the clearest images. These values are only to allow imperative light conditions for imaging to prevent unwanted diffusion or scattering from the light source.

The comparison of the magnification and the focal image positions in the Blu-ray Disc for 30,50 and $100 \mu \mathrm{m}$ PS microspheres is shown in figure 4. In experiments, the super-resolution imaging is lost in out of range graphs. The magnification is increased when the focal image position is 

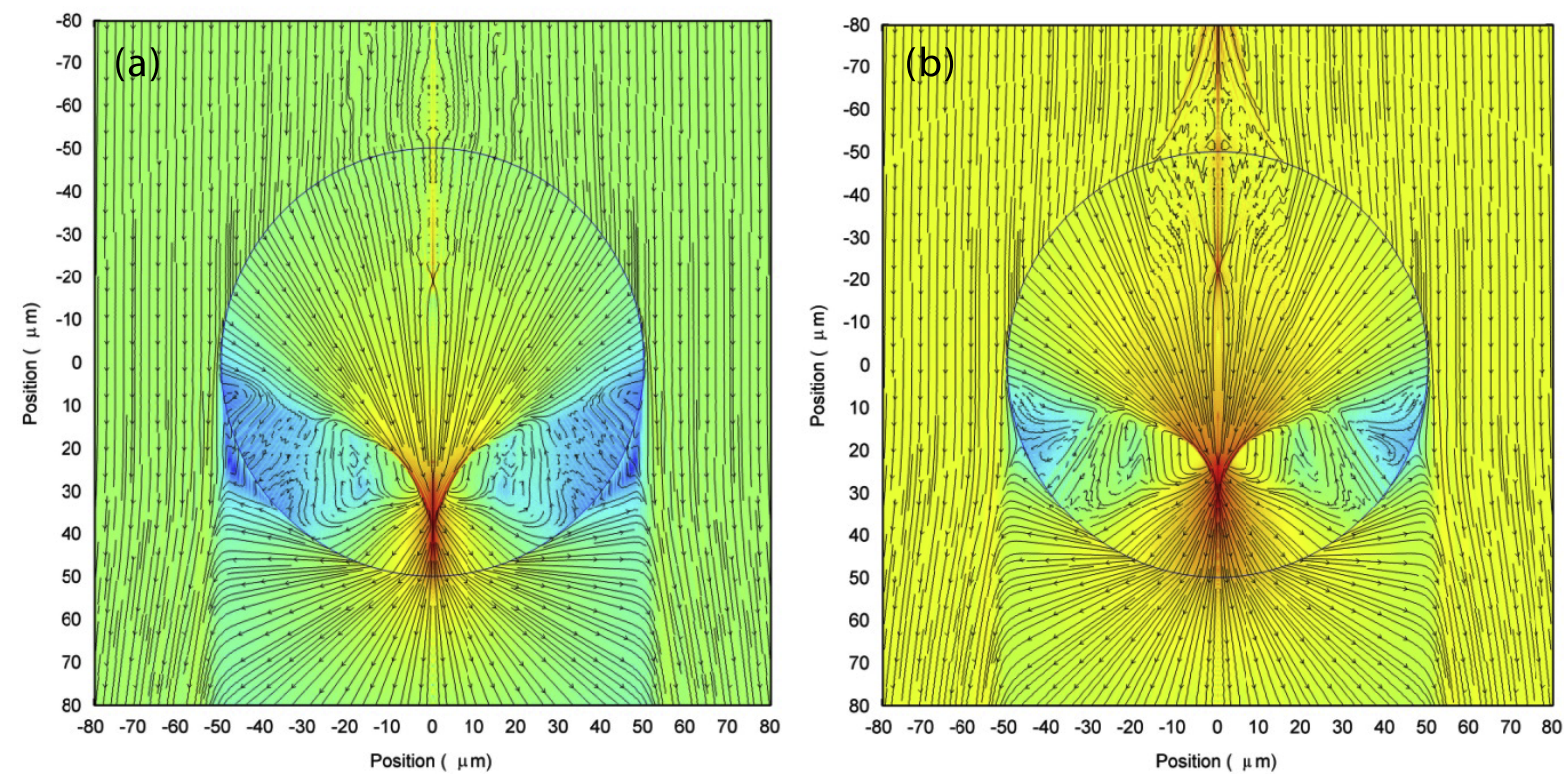

Figure 7. Poynting vector streamlines of the high refractive index microspheres at $600 \mathrm{~nm}$ wavelength. A $100 \mu \mathrm{m}$ diameter microsphere with the refractive index (a) $n=2.2$ and (b) $n=2.6$ is simulated. The highest intensity positions are (a) $38.40 \mu \mathrm{m}$ and (b) $29.12 \mu \mathrm{m}$.

far from the target substrate but the resolution and contrast of the image were rapidly decreased. The image resolution and contrast are good with 50 and $100 \mu \mathrm{m}$ PS microspheres but there is a lack of centre imaging in $30 \mu \mathrm{m}$ PS microspheres because the black spot is continuously observed to disturb imaging. The magnification can depend on the size of the PS microsphere. The $30 \mu \mathrm{m}, 50 \mu \mathrm{m}$ and $100 \mu \mathrm{m}$ magnification at $\alpha=3.0$ is approximately 6.5 times, 7.5 times and 8.0 times, respectively. The good resolution for focal image positions is $100-140 \mu \mathrm{m}$ in $50 \mu \mathrm{m}$ PS microspheres and $260-300 \mu \mathrm{m}$ in $100 \mu \mathrm{m}$ PS microspheres. It should be noted that, as the evanescent wave does not have a diffraction limit, the imaging resolution is very much dependent on how much the images can be magnified to the diffraction limit for the standard optical microscope and pick of the converted images. For this particular optical microscope with diffraction limit of $300 \mathrm{~nm}$, about seven times microsphere magnification would allow the optical microscope to observe $43 \mathrm{~nm}(300 \mathrm{~nm} / 7 \times$ magnification) in an ideal situation if there is no spherical aberration of the objective lens.

Image resolution and focal image positions of $100 \mu \mathrm{m}$ PS microspheres are compared in the Blu-ray Disc as shown in figure 5. Clear super-resolution images are obtained below 260-300 $\mu \mathrm{m}$ focal image position in figures 5(c)-(e). Distorted images are observed in figures 5(a) and (b), and the super-resolution imaging can begin to be lost by blurring in figure 5(f). The magnification is increased when the focal image position is deeper but the super-resolution can be lost below $320 \mu \mathrm{m}$. The loss of resolution may cause the focal position of the object lens to be misaligned with the image position of the microsphere.

The Poynting vector streamlines and the highest intensity positions of 30,50 and $100 \mu \mathrm{m}$ diameter PS microspheres are simulated at $600 \mathrm{~nm}$ visible wavelength using Mie theory calculation as shown in figure 6. For the PS microspheres, the relationship between Poynting vector streamlines and the maximum intensity can provide the procedure of superresolution imaging in the MONS. The scattering and diffusion of the microspheres is not observed during Poynting vector streamlines in 30,50 and $100 \mu \mathrm{m}$ diameter PS microspheres, so EM waves over the microspheres may not interrupt imaging. This means that sufficient energy of imaging can be provided the microspheres, and then this energy can directly deliver to the object lens. Thus, the high intensity energy can involve more information of sub-diffraction-limited features from transforming evanescent waves into propagating waves through the microsphere. Moreover, during light tracing of the microsphere in the near-field, the resonance effect is also enhanced by the conversion of the evanescent wave to the propagating wave [7] and this enhanced effect can encourage the energy of imaging.

The super-resolution imaging of the microsphere can be expected from the focal position and the maximum intensity. The focal position is decided by the relationship between the size and refractive index of dielectric materials $[15,16]$. The high refractive index of dielectric materials can increase the maximum intensity but the focal position may be located inside the dielectric materials. In figure 7, the inside focus from the high refractive index of the microsphere can create scatterings around the microsphere so the imaging of transforming evanescent waves into propagating waves may be disturbed by such optical interruptions. Furthermore, from the super-resolution strength, the super-resolution imaging of the microsphere in air can be restricted by the refractive index $n>2.0$, or the over $10 \mu \mathrm{m}$ diameter of the microsphere with the refractive index $n=1.46$ [12]. For increasing maximum intensity, the curvature of the microsphere can generate higher maximum intensity than the solid immersion lens. For example, the maximum intensity of a fused silica microsphere $\left(I_{\max }=107\right)$ is higher than the same diameter solid immersion lens $\left(I_{\max }=90\right)$ [12]. 


\section{Conclusions}

The MONS can deliver the magnified image of sub-diffraction structures in the reflective EM radiation. In the MONS, a microsphere acts as a very narrow detector that absorbs evanescent waves and transforms evanescent waves into propagating waves. Large microspheres (above $30 \mu \mathrm{m}$ ) can also achieve super-resolution imaging with large view windows in air without immersion liquids. In the 30, 50 and $100 \mu \mathrm{m}$ diameter PS microspheres, the magnification and resolution are affected by the focal image position and the size of the microsphere. The super-resolution was experimentally observed when the optical microscope was focused below double to triple diameters of the microspheres from the target surface in the Blu-ray Disc and the gold nano-patterned quartz. Approximately six to eight times magnification was achieved to resolve sub-diffraction features using the optical microscope.

\section{References}

[1] Zhang X and Liu Z 2008 Superlenses to overcome the diffraction limit Nature Mater. 7 435-41

[2] Pendry J B 2000 Negative refraction makes a perfect lens Phys. Rev. Lett. 85 3966-9

[3] Liu Z, Fang N, Yen T J and Zhang X 2003 Rapid growth of evanescent wave by a silver superlens Appl. Phys. Lett. 83 5184-6

[4] Aydin K, Bulu I and Ozbay E 2007 Subwavelength resolution with a negative-index metamaterial superlens Appl. Phys. Lett. 90254102
[5] Liu Z, Durant S, Lee H, Pikus Y, Fang N, Xiong Y, Sun C and Zhang X 2007 Far-field optical superlens Nano Lett. 7 403-8

[6] Xiong Y, Liu Z, Sun C and Zhang X 2007 Two-dimensional imaging by far-field superlens at visible wavelengths Nano Lett. 7 3360-5

[7] Ben-Aryeh Y 2008 Transmission enhancement by conversion of evanescent waves into propagating waves Appl. Phys. B 91 157-65

[8] Smolyaninov I I, Hung Y J and Davis C C 2007 Magnifying superlens in the visible frequency range Science 315 1699-701

[9] Rho J, Ye Z, Xiong Y, Yin X, Liu Z, Choi H, Bartal G and Zhang X 2010 Spherical hyperlens for two-dimensional sub-diffractional imaging at visible frequencies Nature Commun. 1143

[10] Li J, Fok L, Yin X, Bartal G and Zhang X 2009 Experimental demonstration of an acoustic magnifying hyperlens Nature Mater. 8 931-4

[11] Liu Z, Lee H, Xiong Y, Sun C and Zhang X 2007 Far-field optical hyperlens magnifying sub-diffraction-limited objects Science 3151686

[12] Wang Z, Guo W, Li L, Luk'Yanchuk B, Khan A, Liu Z, Chen $Z$ and Hong M 2011 Optical virtual imaging at $50 \mathrm{~nm}$ lateral resolution with a white-light nanoscope Nature Commun. 2218

[13] Ben-Aryeh Y 2006 Tunneling of evanescent waves into propagating waves Appl. Phys. B 84 121-4

[14] Goodman J W 2005 Introduction to Fourier Optics (Englewood, CO: Roberts \& Company)

[15] Chen Z, Taflove A and Backman V 2004 Photonic nanojet enhancement of backscattering of light by nanoparticles: a potential novel visible-light ultramicroscopy technique $O p t$. Express 12 1214-20

[16] Luk' yanchuk B S and Ternovsky V 2006 Light scattering by a thin wire with a surface-plasmon resonance: bifurcations of the Poynting vector field Phys. Rev. B 73235432 